\title{
MRI to determine the chronological age of Ghanaian footballers
}

\author{
B D Sarkodie, ${ }^{1}$ BSc, MB ChB, FWACS; E K Ofori, ${ }^{2}$ BSc (Hons); MPhil, PhD; P Pambo,${ }^{3}$ BSc, $\mathrm{MSc}, \mathrm{MB} \mathrm{ChB}$ \\ ${ }^{1}$ Radiology Department, Tamale Teaching Hospital, Tamale, Ghana \\ ${ }^{2}$ Department of Radiography, School of Allied Health Sciences, College of Health Sciences, University of Ghana, Korle-Bu-Accra, Ghana \\ ${ }^{3}$ Ghana Football Association, Ghana
}

Corresponding author: B D Sarkodie(ghana_neo@yahoo.com)

\begin{abstract}
Background. The chronological age of the ordinary Ghanaian has often been difficult to verify as registration at birth is not compulsory. Consequently, an accurate method of age determination is needed in competitive age-restricted sports.

Objective. To evaluate the age of Ghanaian soccer players who are aspiring to play for the national under-17 (U17) team, using the degree of fusion of the distal radius on magentic resonance imaging (MRI) and comparing it with the Fédération Internationale de Football Association (FIFA) MRI grading.

Methods. MRI scans of the left wrists of 86 players aspiring to play for the national U17 football team were recruited for the study during a 'justify your inclusion tournament' organised by the Ghana Football Association between June and August 2012. The study was conducted in a diagnostic centre in Accra using a 0.35T MRI scanner. The images were analysed using the previously published FIFA grading system. Results. The mean chronological age of the players was 15.4 years (standard deviation \pm 0.7 ; range 12 - 17). The study showed that $43.0 \%$ of the MRI images were grade 6 ( $\geq 17$ years) in relation to the degree of fusion of the distal radius, and $93.0 \%$ of the grade 6 players were aged 15 - 16 years chronologically. There was no significant correlation between the chronological age and the degree of fusion $(r=0.075 ; p=0.493)$. Conclusion. Ghanaian U17 soccer players seem to be more biologically mature than a normative population of the same age category. The lack of correlation between age category and degree of fusion supports the suspicion that most Ghanaian players may not know their true age.
\end{abstract}

S Afr J SM 2013;25(3):74-76. DOI:10.7196/SAJSM.487

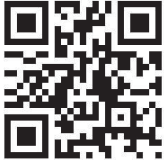

Despite the increasing use of radiological investigations worldwide as a result of their known benefits to society, ${ }^{[1]}$ diagnostic X-rays are by far the largest contributor to the collective dose of all man-made radiation. ${ }^{[2,3]}$ Exposure to such radiation is associated with an increased long-term risk for malignant disease. ${ }^{[4,5]}$ The basic safety standards of the International Atomic Energy Agency (IAEA) for the protection against ionising radiation and safety of radiation sources ${ }^{[6]}$ recommends considering alternative imaging methods (e.g. magnetic resonance imaging (MRI) or ultrasound) if they provide equal or better information. These modalities avoid the use of ionising radiation and the subsequent risks to the patient. ${ }^{[7]}$

Accurate age determination is vital in any competitive agerestricted sport; inconsistencies in age lead to unequal chances and counter both the spirit of the game and 'fair play'. The determination of skeletal maturity has an important place in the practice of paediatrics, especially in relation to endocrinological problems and growth disorders. ${ }^{[8]}$ Standard radiographs of the left wrist are widely used for assessment of skeletal age, although they may vary depending on ethnic origin. ${ }^{[9,10]}$

The need for an alternative method of determining age and maturity has been raised by the IAEA, ${ }^{[9]}$ which does not allow an $\mathrm{X}$-ray examination except when clinically justified for the individual. Consequently, the use of X-rays (a source of radiation exposure) to determine whether or not players are over age, is not encouraged.

Whereas standard radiographs with the attendant radiation risk cannot be justified as the screening tool in soccer populations, age estimation on the basis of grading of fusion of the distal radius by MRI has been shown to be a reliable and valid method in 14 19-year-old soccer players. ${ }^{[11]}$ In Ghana, chronological age has often been a difficult challenge, as registration at birth is not compulsory. This gives room for falsification of true age, which could lead to cheating. ${ }^{[10]}$

It has been suggested that age, experience, body size and stage of puberty contribute considerably, in different combinations, to the variance of some football skills such as dribbling with a pass, ball control with the body and shooting accuracy. ${ }^{[12]}$ Also, players with a greater relative (or possibly false lower) age are more likely to be identified as 'talented' because of the likely physical advantages that they have over their 'younger' peers. ${ }^{[12]}$

We aimed to evaluate the age of aspiring Ghanaian under-17 (U17) footballers using the degree of fusion of the distal radius on MRI and to compare it with Fédération Internationale de Football Association (FIFA) MRI grading.

\section{Methods}

The study was prospective and cross-sectional, employing MRI scans of the left wrist of 86 players aspiring to play for the national U17 football team. Table 1 summarises the characteristics of the MRI equipment used in the study. Participants with a chronological age of $12-17$ years were recruited during a 'justify your inclusion tournament' organised by the Ghana Football Association between June and August 2012. The study was conducted in the Sweden Ghana Medical Centre (SGM) in Accra. The degree of fusion of the left distal 
radial physis was determined by a team comprising two consultant radiologists. The images and their corresponding reports were analysed concurrently using the previously published FIFA grading system. ${ }^{[9,11]}$

\section{Statistical analysis}

Inter-rater agreement was calculated using kappa. ${ }^{[13,14]}$ Kappa values can range from 0 (no agreement) to 1 (total agreement). To measure the inter-rater agreement, a random selection of 20 of the images which had been assessed by the first consultant radiologist were graded blindly by the second consultant radiologist and vice versa. The results were then compared and kappa was calculated using SPSS (version 17) to determine the level of inter-rater agreement. Both descriptive and inferential statistics were carried out using SPSS.

\section{Results}

\section{Age and degree of fusion}

A total of 86 players with self-reported ages of 12 - 17 years presented for the MRI scan of the left wrist. The mean age of the players was 15.4 years ( $\mathrm{SD} \pm 0.7)$. The majority of the players $(80 / 86 ; 93 \%)$ were aged between 15 and 16 years. Table 2 shows the crosstabulation of self-reported chronological age and the degree of fusion. Table 3 summarises the distribution of players according to each grade of fusion.

\section{Inferential analysis}

The self-reported chronological ages according to the six grades of distal radius fusion are presented by the error bar plots in Fig. 1. Mean ages and $95 \%$ CIs were calculated using SPSS (version 17). A scatter plot of self-reported chronological age v. degree of fusion is presented in Fig. 2.

\section{Inter-rater agreement}

Kappa was calculated to determine the level of agreement between the two radiologists. A kappa value of 0.94 (94.0\%) was recorded, indicating very good agreement. However, there was no significant correlation between the chronological age and the degree of fusion $(r=0.075 ; p=0.493)$.

\section{Discussion}

In FIFA U17 competitions, players must have been born on 1 January and/or be $<17$ years old before the year of the tournament, i.e.

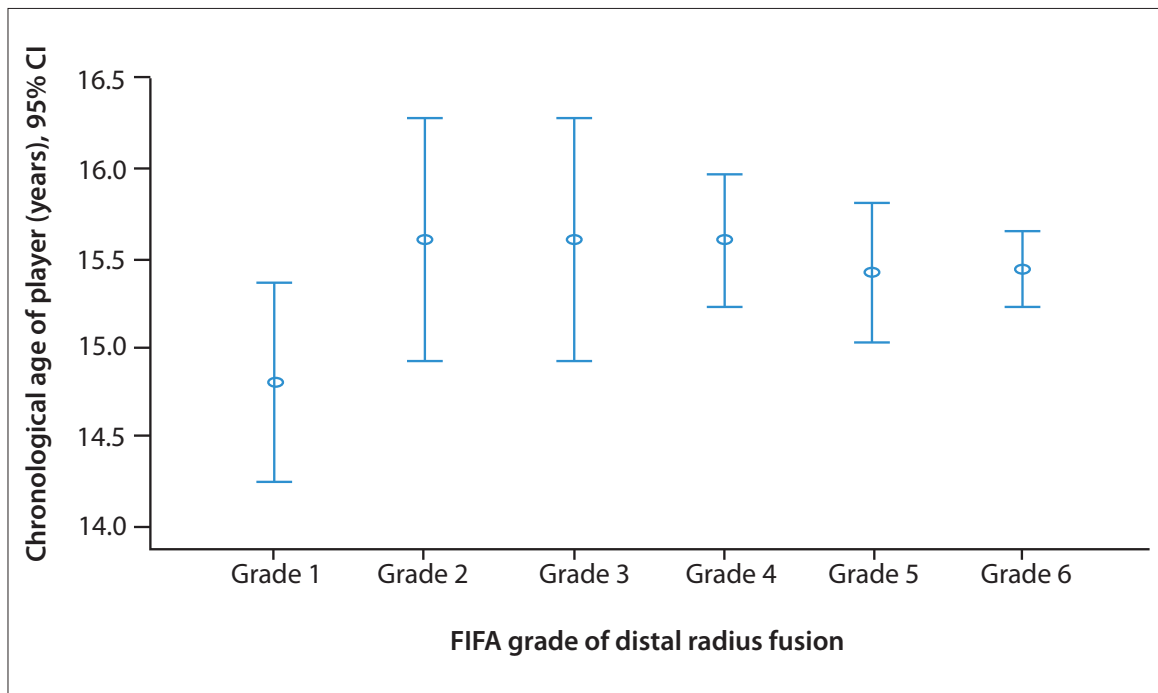

Fig. 1. Error bar plot with 95\% CI for chronological age by degree of fusion.

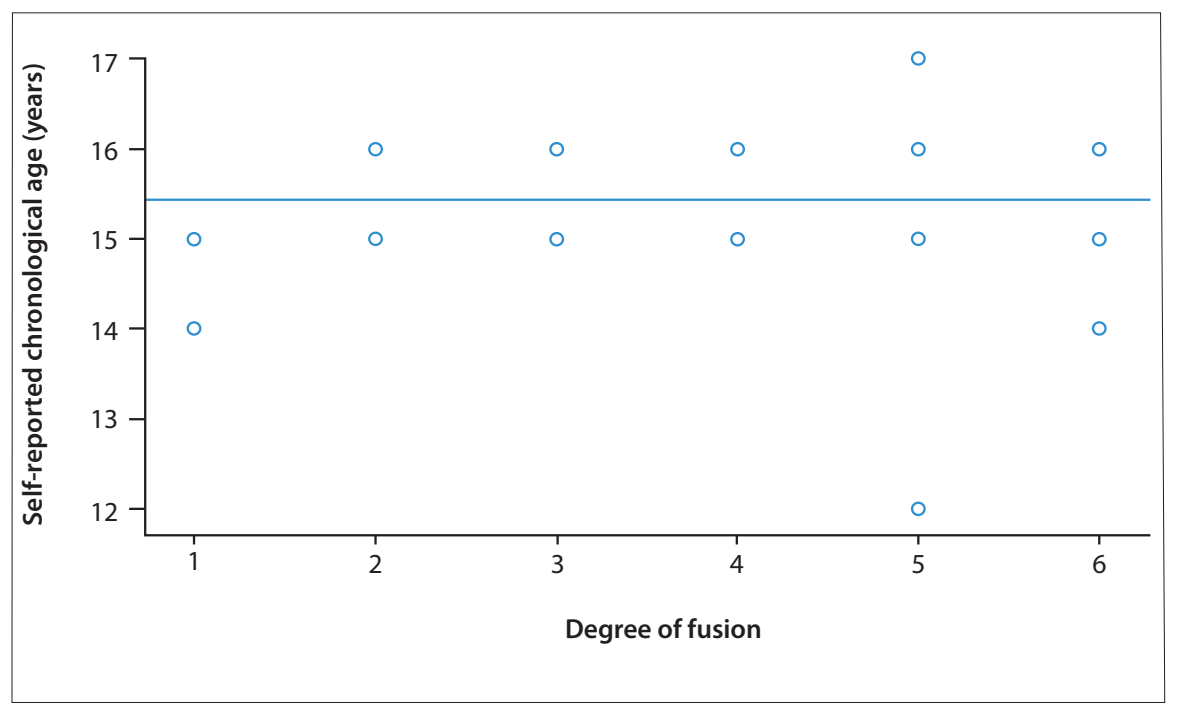

Fig. 2. Scatter plot of self-reported chronological age v. degree of fusion.

the players in FIFA U17 can be aged 17 years at the start of the championship. Because of biological variability, the 'true' age of an individual can only be estimated with a certain probability, but MRI of the wrist has shown to be a valid and a reliable method for estimating age in 14 - 19-year-old soccer players in a normative population. ${ }^{[9]}$ FIFA requires all players to undergo a mandatory MRI test before the regional and U17 world cup tournament. Based on the findings of previous studies, ${ }^{[9,10]}$ the probability of complete fusion occurring prior to 17 years of age is $<1 \%$. In other words, if MRI shows complete fusion of a player's wrist, the player is likely to be older than 17 years with a certainty of $>99 \%$. However, this study did not include any black Africans.
Table 1. Characteristics of the MRI equipment used in the study

\begin{tabular}{ll}
\hline Parameter & Description \\
\hline Scanner make & Siemens \\
Magnet type & Permanent \\
Magnetic field strength & $0.35 \mathrm{~T}$ \\
Type of coil & Wrist \\
$\begin{array}{l}\text { Protocols for left wrist } \\
\text { (bone age determination) }\end{array}$ & Coronal \\
Frequency & $256 \mathrm{~Hz}$ \\
Phase number & 224 \\
NSA & 4 \\
Thickness & $3 \mathrm{~mm}$ \\
Interval & $2.5 \mathrm{~mm}$ \\
Field of view & $180 \mathrm{~mm}$ \\
&
\end{tabular}


Table 2. Cross-tabulation of self-reported chronological age and degree of fusion

\begin{tabular}{lllllll}
\hline & \multicolumn{5}{c}{$\begin{array}{c}\text { Self-reported chronological } \\
\text { age (years) }\end{array}$} & \\
\cline { 2 - 6 } Degree of fusion & $\mathbf{1 2}$ & $\mathbf{1 4}$ & $\mathbf{1 5}$ & $\mathbf{1 6}$ & $\mathbf{1 7}$ & Total \\
\hline Grade 1 & 0 & 1 & 4 & 0 & 0 & 5 \\
Grade 2 & 0 & 0 & 2 & 3 & 0 & 5 \\
Grade 3 & 0 & 0 & 2 & 3 & 0 & 5 \\
Grade 4 & 0 & 0 & 4 & 6 & 0 & 10 \\
Grade 5 & 1 & 0 & 11 & 11 & 1 & 24 \\
Grade 6 & 0 & 3 & 15 & 19 & 0 & 37 \\
Total & 1 & 4 & 38 & 42 & 1 & 86
\end{tabular}

Table 3. Distribution of players in each grade of fusion

\begin{tabular}{lll}
\hline $\begin{array}{l}\text { Degree of } \\
\text { fusion }\end{array}$ & $\boldsymbol{n}(\%)$ & $\begin{array}{l}\text { Self-reported chronological } \\
\text { age (years), mean }( \pm \text { SD) }\end{array}$ \\
\hline Grade 1 & $5(5.8)$ & $14.8( \pm 0.5)$ \\
Grade 2 & $5(5.8)$ & $15.6( \pm 0.6)$ \\
Grade 3 & $5(5.8)$ & $15.6( \pm 0.6)$ \\
Grade 4 & $10(11.6)$ & $15.6( \pm 0.5)$ \\
Grade 5 & $24(27.9)$ & $15.4( \pm 0.9)$ \\
Grade 6 & $37(43.0)$ & $15.4( \pm 0.7)$ \\
Total & $86(100)$ & $15.4( \pm 0.7)$ \\
& &
\end{tabular}

In the present study, the MRI images of $43.0 \%(n=37)$ of the aspiring players were graded 6 , i.e. completely fused or over-age, which is substantially higher compared with the findings of other studies in Europe and Asia. ${ }^{[9,10]}$ The highest number of players with complete fusion (Grade 6) was found among those with a chronological age of 16 years. Approximately three-quarters (75\%) of the players aged 15 and 16 years were over-age (Grade 6). From the MRI results, the aspiring players seemed to be more mature than a normative population of soccer players.

No significant correlation was observed between age category and grade of fusion $(r=0.075 ; p=0.493)$. This finding is consistent with previous studies $(p=0.13) .^{[9,10]}$

The missing correlation between the age presented by participants and biological maturity assessed from fusion of the distal radius cannot be explained definitively. This discrepancy may support the suspicion that some U17 players are older than stated in their official documents; however, ethnic variations may also account for the difference between chronological age and the degree of fusion on MRI. It is reported that prepubertal American children of European descent have significantly delayed skeletal maturation compared with those of African descent, and postpubertal American children of European descent have significantly advanced skeletal maturation compared with postpubertal children of African descent. ${ }^{[15]}$

\section{Conclusion}

The use of MRI to investigate the degree of fusion of the left distal radius has given an indication of the maturity of aspiring $\mathrm{U} 17$ soccer players in Ghana. On the basis of the findings, the self-reported ages of these players might not have been correct compared with results from normative studies, although other factors may be responsible for the differences.

Accurate age determination of participants is important in competitive sport. However, predicting age using the degree of fusion of the left distal radius of the wrist as a method to verify chronological age may lack the accuracy needed and may result in many false-positive or false-negative results, until a normative study is carried out on black Africans. Registration at birth is not compulsory in many African countries such as Ghana; therefore, it has been argued that a normative study may be difficult to carry out. However, there are various ways to verify the ages of 14 - 19-year-olds, especially in a situation where there is no incentive for one to hide their true age. There is an urgent need for a comprehensive study involving black Africans to ensure that the black African footballer is given a fair playing ground by the FIFA.

Acknowledgements. We acknowledge the support and co-operation of the staff of the Sweden Ghana Medical Centre (SGM) in Accra, Ghana.

\section{References}

1. Saunders M, Budden A, Maclver F. Dose implications of fluoroscopy-guided positioning (FGP) for lumbar spine examinations prior to acquiring plain film radiographs. $\mathrm{Br}$ Radiol 2005;78:130-134. [http://dx.doi.org/10.1259/bjr/50195548]

2. UNSCEAR. Report to the General Assembly, with Scientific Annexes. New York: United Nations, 2000.

3. Manning D. The risk of cancer from radiography. J Radiography 2004;10(3):171-172. [http://dx.doi.org/10.1016/j.rad.2004.02.014]

4. Berrington de Gonzalez A, Darby S. Risk of cancer from diagnostic x-rays: Estimates for the UK and 14 other countries. Lancet 2004;363(9406):345-351. [http://dx.doi. org/10.1016/S0140-6736(04)15433-0

5. Engel-Hills P. Radiation protection in medical imaging. J Radiography 2006;12(2):153160.

6. International Atomic Energy Agency (IAEA). Radiological Protection for Medica Exposure to Ionizing Radiation. IAEA Safe Standard Series 2002. Safety Guide No. RSG-1.5. Vienna: IAEA, 2002.

7. Valentine J. Avoidance of radiation injuries from medical interventional procedures. Ann ICRP 2000;30(2):7-67.

8. Tanner JM, Whitehouse RH, Marshall WA, et al. Prediction of adult height from height, bone age, and occurrence of menarche, at ages 4 to 16 with allowance for mid parent height. Arch Dis Child 1976;50(1):14-26.

9. Dvorak J, George J, Junge A, Hodler J. Application of MRI of the wrist for age determination in international U-17 soccer competition. Br J Sports Med 2007;41(8):497500. [http://dx.doi.org/10.1136/bjsm.2006.033431]

10. Braude SC, Henning LM, Lambert MI. Accuracy of bone assessments for verifying age in adolescents - application in sport. South African Journal of Radiology 2007;11(2):4

11. Dvorak J, George J, Junge A, Hodler J. Age determination by magnetic resonance imaging of the wrist in adolescent male football players. Br J Sports Med 2007;41(1):4552. [http://dx.doi.org/10.1136/bjsm.2006.031021]

12. Malina RM, Eisenmann JC, Cumming SP, et al. Maturity-associated variation in the growth and functional capacities of youth football (soccer) players 13 - 15 years. Eur J Appl Phsyiol 2004;91(5):555-562. [http://dx.doi.org/10.1007/s00421-003-0995-z]

13. Meltzoff J. Criteria and Criteria Measure, Critical Thinking About Research. Washington DC: American Psychological Association, 1998.

14. Bryman A. Social Research Methods. 3rd Ed. Oxford: Oxford University Press, 2008:265.

15. Mora S, Boechat MI, Pietka E, Huang HK, Gilsanz V. Skeletal age determinations in children of European and African descent: Applicability of the Greulich and Pyle standards. Pediatric Res 2001;50(5):624-628. 